\title{
Detection of 2,4-Dinitrophenol and Bio-Imaging Application with Agaric-Based Nitrogen-Doped Carbon Quantum Dots (N-CQDs)
}

\author{
Jiao Zhao, ${ }^{\oplus a, b}$ Xianhao Long, ${ }^{c}{\text { Zhihua } \mathrm{Li}^{d} \text { and Yujiao Tu }}^{*, a}$ \\ ${ }^{a}$ Faculty of Chemistry and Chemical Engineering, Kunming University, 650214 Kunming, Yunnan Province, China \\ ${ }^{b}$ Yunnan Key Laboratory of Food-Safety Testing Technology, Kunming University, 650214 Kunming, \\ Yunnan Province, China \\ 'Department of Postgraduates, Kunming University, 650214 Kunming, Yunnan Province, China \\ 'Yunnan Institute of Nuclear Industry Geological Survey, 650106 Yunnan Province, China
}

\begin{abstract}
With agaric as carbon source and urea as nitrogen source, this paper has successfully synthesized nitrogen-doped carbon quantum dots (N-CQDs) by simple one-step hydrothermal method. A series of analysis have been used to make characterization of the physical, chemical structures and optical performance of N-CQDs. The fluorescence stability was studied by examining the ionic strength, $\mathrm{pH}$ value and storage time and other conditions. The cytotoxicity and bacterial toxicity of N-CQDs were applied to biological imaging. Considering the quenching effect of 2,4-dinitrophenol (2,4-DNP) on the fluorescence generated by N-CQDs, N-CQDs have been used as fluorescence probe in detection of 2,4-DNP where N-CQDs showed high sensitivity, selectivity and strong disturbance resistance to 2,4-DNP based on the quenching mechanism of transfer of resonance energy. It can also be concluded that agaric-based N-CQDs can be used in bio-imaging due to a good inhibitory effect on but low toxicity to bacteria. Under the optimal experimental conditions, the probe has presented a good coefficient of determination $\left(\mathrm{R}^{2}=0.9969\right)$ and a low limit of detection $(15.78 \mathrm{nM})$. This method has been successfully applied to determine 2,4-DNP in environmental samples and provided a new idea for developing green and natural composite carbon materials.
\end{abstract}

Keywords: carbon quantum dots, agaric, 2,4-dinitrophenol, biological imaging

\section{Introduction}

The desire to look attractive is universal. Many young people are trying everything possible to lose weight and keep fit. In 1930s, 2,4-dinitrophenol (2,4-DNP) was used as an oral medicine to control body weight as research showed that it could significantly improve basal metabolic rate, but was soon forbidden to be used for the purpose of losing weight by the Food and Drug Administration due to its serious side effects including hyperthermia, cataract and death. ${ }^{1,2}$ So far, there are 62 death cases attributing to 2,4-DNP in published medical literature. ${ }^{3}$ Therefore, it is of great significance to establish a rapid, simple and highselectivity analysis method for monitoring trace 2,4-DNP in biological and environmental samples and avoiding the occurrence of adverse events.

At present, several methods for determining 2,4-DNP have been reported such as high performance liquid

*e-mail: 88937377@qq.com chromatography (HPLC), ${ }^{4}$ gas chromatography $(\mathrm{GC})^{5}$ and electrochemical sensor. ${ }^{6}$ The GC method, though with good sensitivity and accuracy, is not applicable to conventional analysis in a non-professional laboratory due to high equipment cost and high requirement on proficiency of operators and the electrochemical sensor method cannot avoid interference from substances with similar structure due to incapacity of effective separation. As far as we know, due to lack of selectivity, it is necessary to choose more selective and more effective sample pretreatment to determine 2,4-DNP.

As a novel carbon material, carbon quantum dots (CQDs) have received extensive attention since they were first discovered in 2004. ${ }^{7,8}$ Compared with conventional organic dye materials and semiconductor quantum dots, CQDs are a more excellent luminescent nanometer material with low toxicity, good biocompatibility and high light intensity, tenability and light stability. ${ }^{9,10}$ Therefore, CQDs have been widely applied in catalysis, ${ }^{11}$ biosensing, ${ }^{12}$ bioimaging, ${ }^{13,14}$ drug delivery, ${ }^{15,16}$ optoelectronic device 
and other fields. Based on the change in fluorescence intensity during detection, CQDs can be used to detect $\mathrm{Ag}^{+},{ }^{17} \mathrm{Hg}^{2+},{ }^{18} \mathrm{Cu}^{2+},{ }^{19}$ biological mercaptan, ${ }^{20} \mathrm{NO}^{2-},{ }^{21} \mathrm{PO}_{4}{ }^{3-}, 22$ $\mathrm{ClO}^{-},{ }^{23} \mathrm{CN}^{-},{ }^{24}$ glucose, ${ }^{25}$ dopamine, ${ }^{26}$ thrombin, ${ }^{27} \mathrm{H}_{2} \mathrm{O}_{2},{ }^{28}$ melamine $^{29}$ and deoxyribonucleic acid (DNA). ${ }^{30}$ To our knowledge, however, CQDs have never been used as a fluorescence probe on any 2,4-DNP detection platform. This paper reports a 2,4-DNP sensor based on fluorescence quenching of CQDs which has a broad application prospect in development of 2,4-DNP sensors.

In this paper, a simple, sensitive and environmentally friendly biological sensor has been established, with nitrogen-doped carbon quantum dots (N-CQDs) for detecting 2,4-DNP. It is interesting that 2,4-DNP may quench selectively the fluorescence of CQDs. Therefore, a novel, simple CQD fluorescence probe has been developed. This paper has for the first time applied CQDs to detecting 2,4-DNP and this method has the advantages of high sensitivity, good selectivity and simple and convenient operation. Besides, raw materials for preparing N-CQDs without toxicity make the fluorescence probe more environmentally friendly. What is more important, this method is of great potential application value for detection of 2,4-DNP in environmental samples.

\section{Experimental}

Instruments and reagents

Agarics were purchased from a local supermarket in Kunming (Yunnan, China); carbamide, citric acid, disodium hydrogen phosphate $\left(\mathrm{Na}_{2} \mathrm{HPO}_{4}\right)$ and sodium hydroxide $(\mathrm{NaOH})$ were purchased from Tianjin Zhiyuan Chemical Reagent Co., Ltd. (Tianjin, China). Metal salts, mainly including $\mathrm{AlCl}_{3} \cdot 6 \mathrm{H}_{2} \mathrm{O}, \mathrm{K}_{2} \mathrm{Cr}_{2} \mathrm{O}_{7}, \mathrm{MgCl}_{2} \cdot 6 \mathrm{H}_{2} \mathrm{O}$, $\mathrm{ZnCl}_{2}, \mathrm{CuCl}_{2} \cdot 2 \mathrm{H}_{2} \mathrm{O}, \mathrm{CdCl}_{2}, \mathrm{PbCl}_{2}, \mathrm{CrCl}_{3}, \mathrm{CoCl}_{2} \cdot 6 \mathrm{H}_{2} \mathrm{O}$, $\mathrm{SnCl}_{2} \cdot 2 \mathrm{H}_{2} \mathrm{O}, \mathrm{FeCl}_{3} \cdot 6 \mathrm{H}_{2} \mathrm{O}$ and $\mathrm{FeCl}_{2} \cdot 4 \mathrm{H}_{2} \mathrm{O}$, were used as analytic reagents. All solutions were prepared with ultrapure water from Milli-Q system $\left(18.2 \mathrm{M} \Omega \mathrm{cm}, 25^{\circ} \mathrm{C}\right)$.

G9800A fluorospectro photometer (Agilent Technologies, USA) was used to determine fluorescence spectrum and fluorescence intensity; UV-2600 ultraviolet-visible spectrophotometer (Shimadzu, Japan) was used to determine ultraviolet-visible spectrum; Tecnai G2F30S-Twin highresolution field emission transmission electron microscope (Tecnai G2 F30S, FEI, Netherlands) was used to detect the particle size and morphology features of N-doped fluorescent quantum dots; TENSOR27 Fourier transform infrared spectrometer (Bruker, Germany) was used to determine infrared spectrum and material structure; D8advance X-ray powder diffractometer (Bruker, Germany) was used to determine crystal structural morphology; Thermo Scientific K-Alpha X-ray photoelectron spectrometer (Thermo Fisher Scientific Inc., USA) was used to analyze the composition ratio and chemical oxidization state of elements; XH-B vortex mixer (Shanghai Hanuo Instrument Co., Ltd., China) was used for vortex mixing; 80-2 highspeed centrifuge (Shanghai Medical Instruments (Group) Ltd., Corp. Surgical Instruments Factory, China) was used for centrifugal filtration; and BPZ-6003 vacuum drying oven (Shanghai Yiheng Instruments Co., Ltd., China) was used for vacuum drying.

\section{Synthesis of agaric-based N-CQDs}

The agarics were set to air dry at room temperature and ground into fine powder. $5 \mathrm{~g}$ powder were weighed and mixed with $3.5 \mathrm{~g}$ carbamide in $90 \mathrm{~mL}$ hot water $\left(60^{\circ} \mathrm{C}\right)$ for ultrasonic dissolution. The solution was transferred into a $200 \mathrm{~mL}$ polytetrafluoroethylene reactor and heated in a muffle at $180^{\circ} \mathrm{C}$ for $5 \mathrm{~h}$ and, after reaction, allowed to cool down naturally to room temperature. The solution was filtered with $0.22 \mu \mathrm{m}$ microfiltration membrane and centrifuged at $13000 \mathrm{rpm}$ for $20 \mathrm{~min}$ to eliminate impurities and get the solution of CQDs. The solution of CQDs was stored at $4{ }^{\circ} \mathrm{C}$ for future use. Synthetic reaction of agaricbased CQDs is as shown in Figure 1.
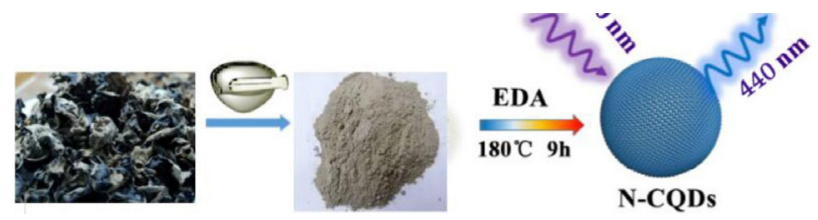

Figure 1. Reaction scheme of synthesis of agaric-based N-CQDs.

\section{Measurement of fluorescence quantum yield}

The fluorescence quantum yield (QY) of the as-prepared agaric-based N-CQDs was measured with quinine sulfate. Quinine sulfate with QY $=0.54$ was dissolved in $0.1 \mathrm{M}$ $\mathrm{H}_{2} \mathrm{SO}_{4}$ (solvent refraction index $(\eta)=1.33$ ) while agaricbased N-CQDs were dissolved in deionized water. The QY was calculated based on the following equation:

$$
\mathrm{Q}=\mathrm{Q}_{\mathrm{S}} \times \frac{\mathrm{A}_{\mathrm{S}}}{\mathrm{A}_{\mathrm{X}}} \times \frac{\mathrm{I}_{\mathrm{X}}}{\mathrm{I}_{\mathrm{S}}} \times\left(\frac{\eta_{\mathrm{X}}}{\eta_{\mathrm{S}}}\right)^{2}
$$

where $\mathrm{Q}$ is quantum yield, A represents the absorbance at the excitation wavelength, I and $\eta$ represent the integrated emission intensity and the solvent refraction index, respectively. The subscript " $S$ " refers to quinine sulfate and " $\mathrm{X}$ " refers to the agaric-based N-CQDs. The QY of agaric-based N-CQDs was calculated to be $30.2 \%$. 

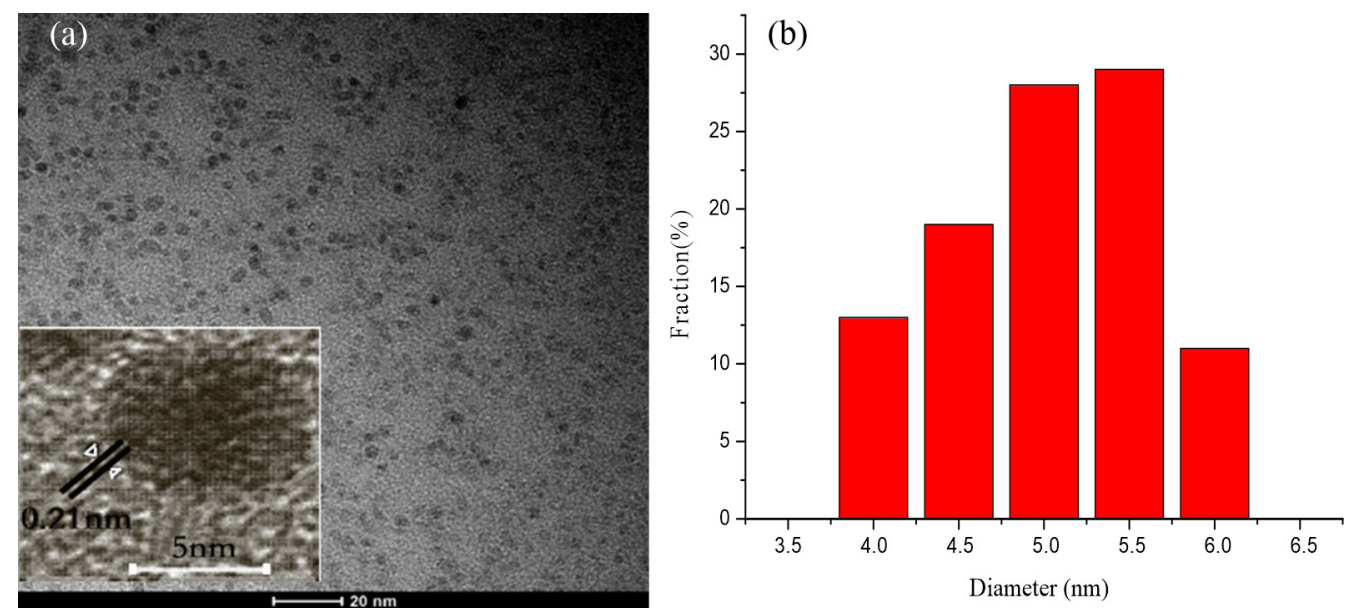

Figure 2. (a) Typical TEM image of N-CQDs. The inset displays the TEM image. (b) Histogram of the size distribution of N-CQDs obtained from TEM image analysis.

\section{Fluorescence quenching test of 2,4-DNP}

$50 \mu \mathrm{L}$ of prepared agaric-based CQDs solution were transferred to a $10 \mathrm{~mL}$ centrifuge tube and diluted to $3 \mathrm{~mL}$ with citric acid- $\mathrm{Na}_{2} \mathrm{HPO}_{4}$ buffer solution for vortex mixing, different concentration 2,4-DNP solution was added, and then diluted to $4.0 \mathrm{~mL}$ with deionized water. The fluorescence intensity at $360 \mathrm{~nm}$ excitation wavelength and $440 \mathrm{~nm}$ emission wavelength was measured after $10 \mathrm{~min}$ reaction at room temperature. As for the fluorescence quenching rate (F/F0) in the calculation system, F0 refers to the fluorescence intensity of the prepared CQDs and $\mathrm{F}$ to the fluorescence intensity after addition of 2,4-DNP solution.

\section{Bioimaging experiment}

Cell culture: culture human colonic adenocarcinoma cells (HT29) line in $37{ }^{\circ} \mathrm{C} 5 \% \mathrm{CO}_{2}$, with $10 \%$ fetal calf serum were added into the Dulbecco's Modified Eagle Medium (DMEM) culture medium. Synthesis of cell-attached slides: the cell-attached slides were put on a 24-hole plate after disinfection and sterilization and the HT29 cell line was inoculated in the 24-hole plate at 20000 hole $^{-1}$; after culture for $24 \mathrm{~h}$ and starvation for one hour, the prepared sample was added at $200 \mu \mathrm{L} \mathrm{hole}^{-1}$ for reaction at $37^{\circ} \mathrm{C}$ for $2 \mathrm{~h}$. Cell fixation: the cells with $4 \%$ paraformaldehyde were fixed at room temperature for $30 \mathrm{~min}$.

\section{Results and Discussion}

\section{Characterization analysis of agaric-based N-CQDs}

To further study the nano-structural characteristics of the synthesized agaric-based N-CQDs, transmission electron microscope (TEM) was used to characterize the microstructure and particle size thereof. As shown in Figure 2a, the synthesized agaric-based N-CQDs are spheroidal carbon nanoparticles uniformly distributed without agglomeration. The sizes of N-CQDs are mainly distributed in the range of 4-7 $\mathrm{nm}$ with a mean diameter of $5 \mathrm{~nm}$, as judged from over 100 individual particles (Figure 2b). The inset of Figure 2a shows the well-resolved lattice fringes with an average interplanar spacing of $0.21 \mathrm{~nm}$, which is very close to the (100) lattice spacing of graphite $\left(\mathrm{d}_{100}=0.21 \mathrm{~nm}\right)$.

The functional group of agaric-based N-CQDs has been studied by Fourier transform infrared spectroscopy (FTIR). As shown in Figure 3, the strong peak at $1490 \mathrm{~cm}^{-1}$ was attributed to symmetric tensile vibration of carboxylic acid ions and that at $3000-3500 \mathrm{~cm}^{-1}$ to tensile vibration of hydroxyl and $\mathrm{N}-\mathrm{H}$, which indicates that carboxylic acid and hydroxyl are the main functional groups on the surface of agaric-based N-CQDs. For 2,4-DNP, the frequency band at $1174 \mathrm{~cm}^{-1}$ corresponds to tensile vibration of $\mathrm{C}-\mathrm{O}$ and $\mathrm{C}-\mathrm{N}$.

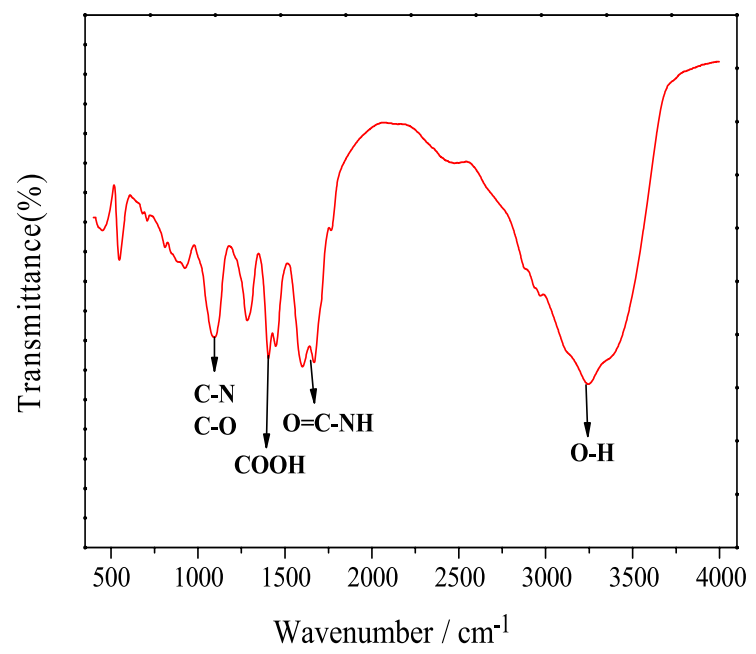

Figure 3. FTIR spectrum of agaric-based N-CQDs. 
The compositions and surface chemical structure of agaric-based N-CQDs have been studied by X-ray electron spectroscopy (XPS). It can be seen from Figure 4 that three peaks at around 287.8, 403.6 and $532.3 \mathrm{eV}$ are presented in the XPS survey spectrum and correspond to C1s, N1s and $\mathrm{O} 1 \mathrm{~s}$, respectively. The results of XPS element analysis are consistent with those obtained by FTIR, demonstrating the existence of oxygen and nitrogen-bearing functional groups such as hydroxyl, carboxyl and amino.

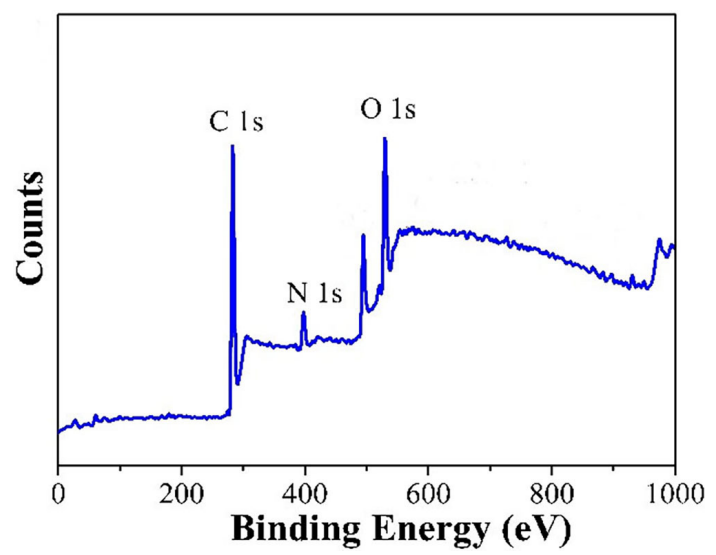

Figure 4. XPS spectra of agaric-based N-CQDs.

\section{Optical properties of agaric-based N-CQDs}

UV-Vis absorption spectra and fluorescence emission spectra have been used to characterize the optical properties of agaric-based N-CQDs. As shown in Figure 5a, there is an obvious absorption peak at $280 \mathrm{~nm}$, which may attribute to the $\mathrm{n}-\pi^{*}$ transition of $\mathrm{sp}^{2}$ structure of $\mathrm{C}=\mathrm{O}$ or $\mathrm{C}-\mathrm{N}$ key. Besides, it can be seen from the inset of Figure 5a that agaric-based N-CQDs appear white under natural light (right) and emit blue luminescence under $365 \mathrm{~nm}$ UV-light (left). Figure $5 \mathrm{~b}$ shows the fluorescence emission spectra of agaric-based N-CQDs at different excitation wavelengths which present a good fluorescence behavior depending on excitation wavelength. The results show that nitrogen doping can adjust the inherent electronic and surface properties of CQDs and improve the effective surface defects and carbon nucleus structure, thus significantly improve the optical properties thereof.

The fluorescent lifetime of the solution of agaric-based $\mathrm{N}-\mathrm{CQDS}$ and that of agaric-based N-CQDs mixed with 2,4-DNP has been determined by time-correlated single photon counting (TCSPC). As the fluorescent lifetime of a fluorescent substance relates to its structure, the polarity of the microenvironment it is located in, the velocity and other conditions, the changes in the system under study can be directly acquainted through determination of fluorescent lifetime. Fluorescence phenomenon occurs
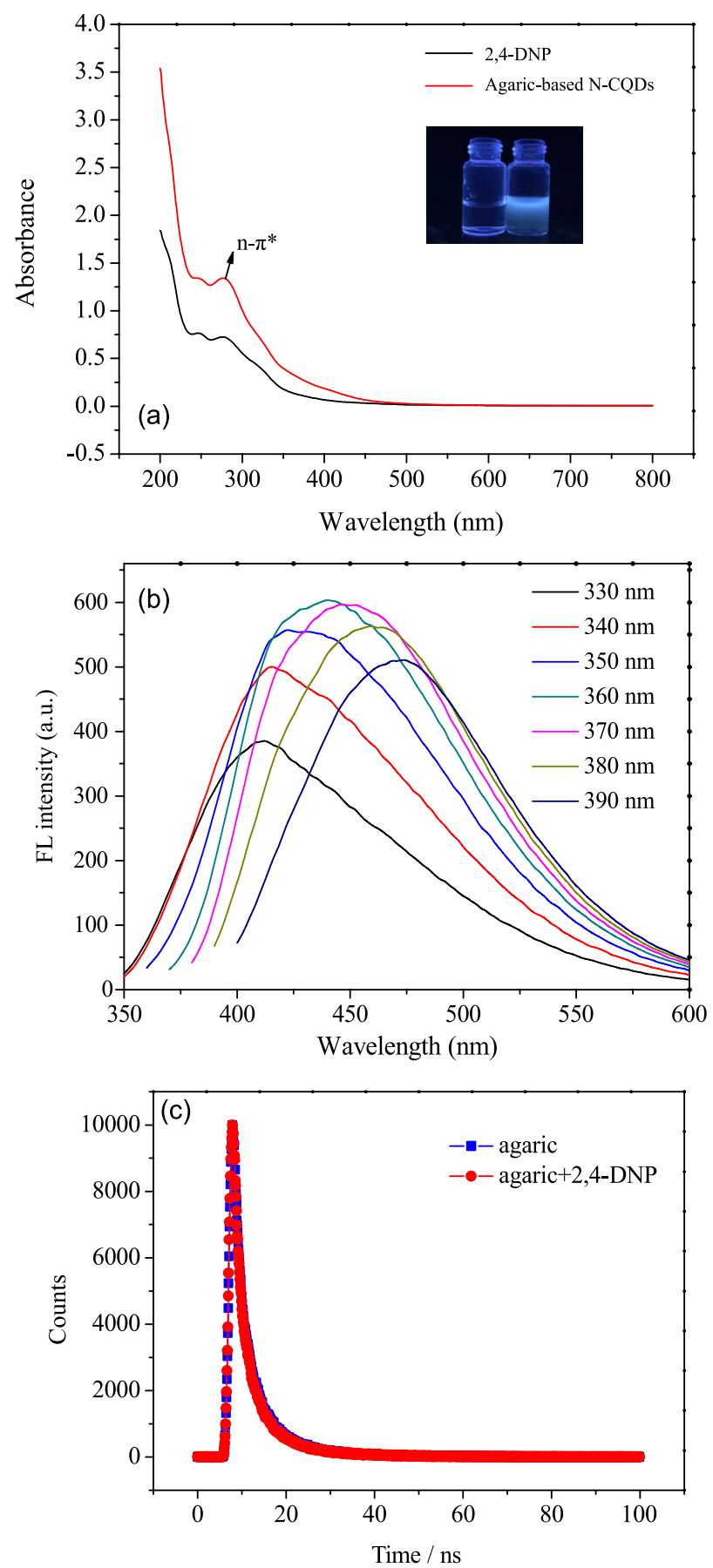

Figure 5. (a) UV-Vis absorption spectra of agaric-based N-CQDs and 2,4-DNP (inset: photographs under natural light (left) and $365 \mathrm{~nm}$ UV light (right)); (b) fluorescence emission spectra of agaric-based N-CQDs at different excitation wavelengths; (c) curves of fluorescent lifetime attenuation of the solution of agaric-based N-CQDs and that of agaricbased N-CQDs + 2,4-DNP.

mostly at the level of nanosecond which is exactly the time scale for occurrence of molecular movement, so the interaction among many complex molecules can be "seen" by virtue of fluorescent technique, such as cluster among molecules in the supramolecular system, conformation rearrangement of absorbed-state macromolecules on the 
solid-fluid interface and changes in higher-order structure of protein. In addition to direct application, determination of fluorescent lifetime is also the basis for other time-resolved fluorescence techniques, e.g., fluorescence quenching technique, which can be used to study the difficulty for quencher and fluorescence indicator or probe to get close to each other, thus enabling judgment on the properties of the microenvironment where the indicator or probe of the system under study is located in, and time resolved fluorescence spectra (FS), which can be used to study the intramolecular action or intermolecular interaction at the speed thereof. None of the major fluorescent techniques including non-radiative energy transfer and time-resolved fluorescence anisotropy can be separable from determination of fluorescent lifetime. In material research, the energy level structure and such information as excited-state relaxation time of a material can be acquired through determination of its fluorescent lifetime. The mean of the two values of fluorescent lifetime obtained from the experiment is $8.0078 \mathrm{~ns}$ and Figure $5 \mathrm{c}$ shows the curves of lifetime attenuation.

\section{Light stability test of agaric-based N-CQDs}

To explore the capacity of agaric-based N-CQDs to resist disturbance from external environment, light stability test has been carried out to investigate ion strength, $\mathrm{pH}$, storage time and other conditions. As shown in Figure 6a, the fluorescence intensity of citric acid-disodium hydrogen phosphate buffer solution remains unchanged when its $\mathrm{pH}$ increases from 2.2 to 8.0 , which indicates that the probe can be used as a fluorescent nanoprobe in acid and alkaline media. It can be seen from Figure $6 \mathrm{~b}$, which shows the effect of ion strength upon the fluorescence intensity of agaric-based N-CQDs, that no fluctuation occurs to the fluorescence intensity with gradual rise in the concentration of $\mathrm{NaCl}$ solution, indicating strong stability of agaric-based $\mathrm{N}-\mathrm{CQD}$ even in an environment with high ion strength. As shown in Figure 6c, under the same test conditions, no obvious change occurs to the fluorescence intensity of agaric-based N-CQDs with the same concentration after placed at room temperature for a period of time. The above test results demonstrate the excellent light stability of the synthesized agaric-based N-CQDs.

\section{Selectivity test}

To explore the selectivity of the fluorescence intensity of agaric-based N-CQDs to the response of different substances or ions, a selectivity test has been carried out for 8 phenols with the same concentration,
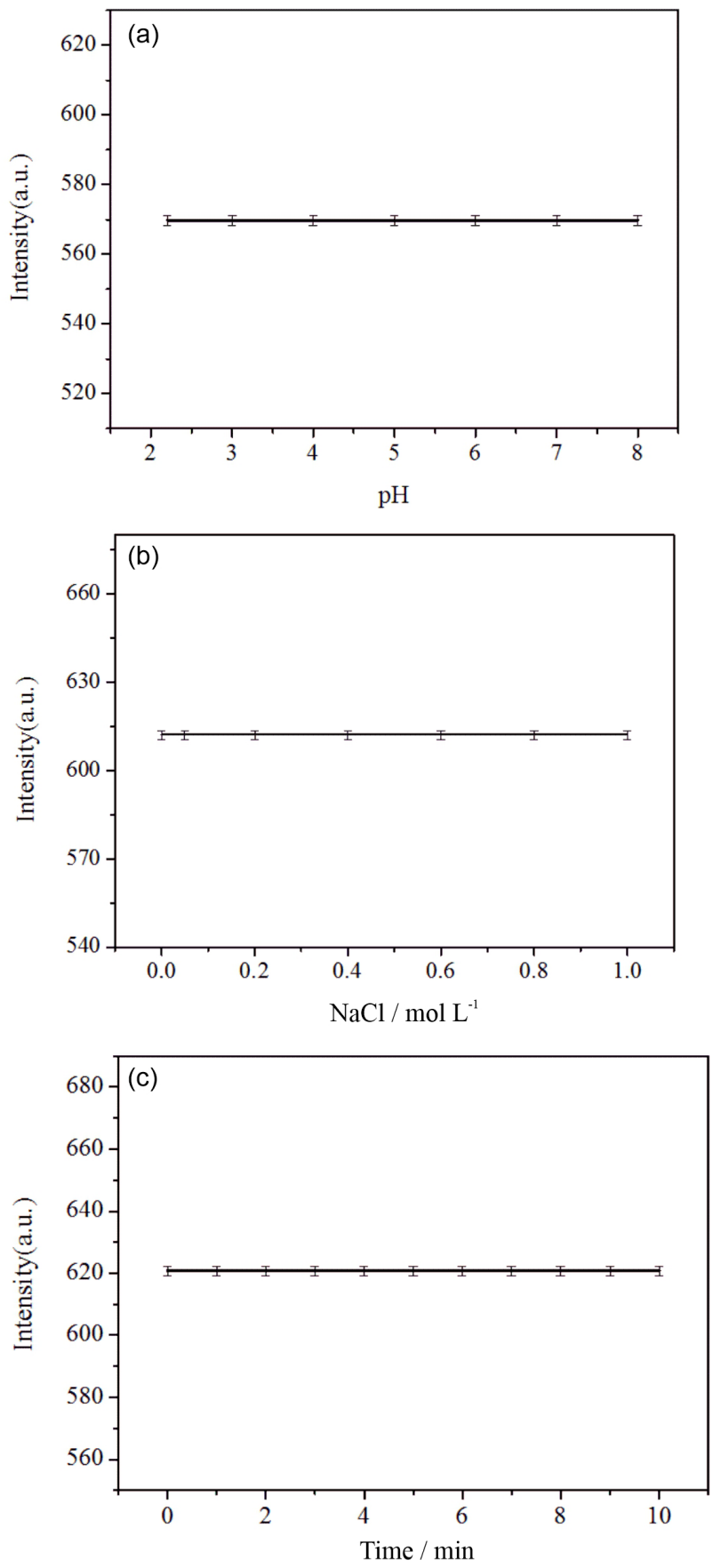

Figure 6. Effects of various conditions on the fluorescence intensity of agaric-based N-CQDs: (a) $\mathrm{pH}$; (b) $\mathrm{NaCl}$ concentration; and (c) storage time, and variation of fluorescence intensity of agaric-based N-CQDs under $365 \mathrm{~nm}$ UV light.

i.e., nonyl phenol, octyl phenol, 2,4,6-trichlorophenol, 2,4-dichlorophenol, parachlorophenol, phenol, 2,4-DNP, paranitrophenol and blank. As shown in Figure 7, except 2,4-DNP which can effectively quench the fluorescence intensity of agaric-based N-CQDs, none of the other phenols have led to any obvious change in the fluorescence intensity of agaric-based N-CQDs, indicating that agaricbased N-CQDs can be used as a nanoprobe to detect 
2,4-DNP through fluorescence quenching due to its high selective response thereto.

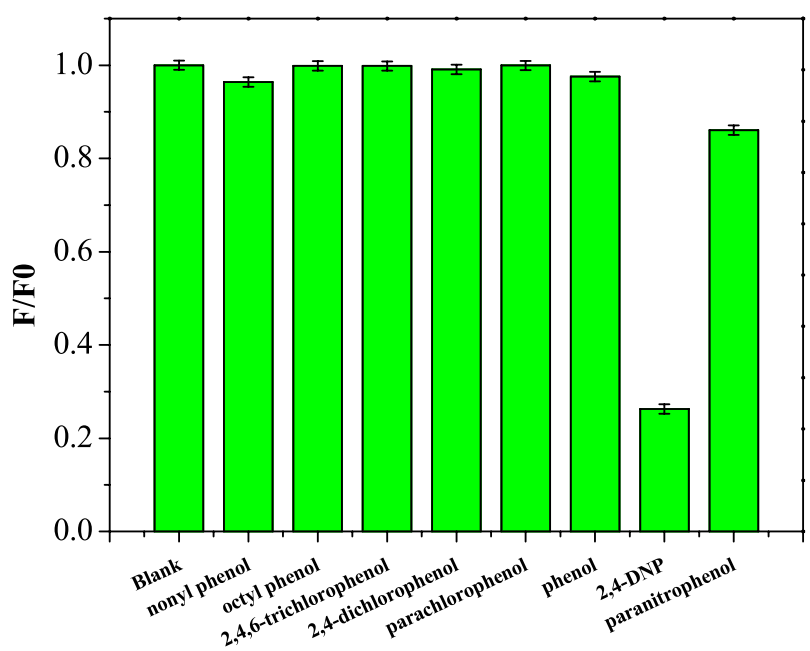

Figure 7. Fluorescence quenching effect of different phenols.

\section{Optimization of experiment conditions}

Based on the applicability of fluorescence quenching mechanism to rapid detection of 2,4-DNP, key experimental factors like $\mathrm{pH}$ of solution and reaction time have been observed and optimized to get the optimal fluorescent response signal. A research has been first made on the change in fluorescence intensity of agaric-based N-CQDs within the $\mathrm{pH}$ range of 2.0-8.0. It can be seen from Figure 8 that the fluorescence quenching efficiency reaches its maximum at $\mathrm{pH}$ 6.0. The influence of $\mathrm{pH}$ value on the quenching process may be related to the acid-base balance group on the surface of agaric-based N-CQDs. ${ }^{31}$ Therefore, $\mathrm{pH} 6.0$ is defined as the optimal determination condition.

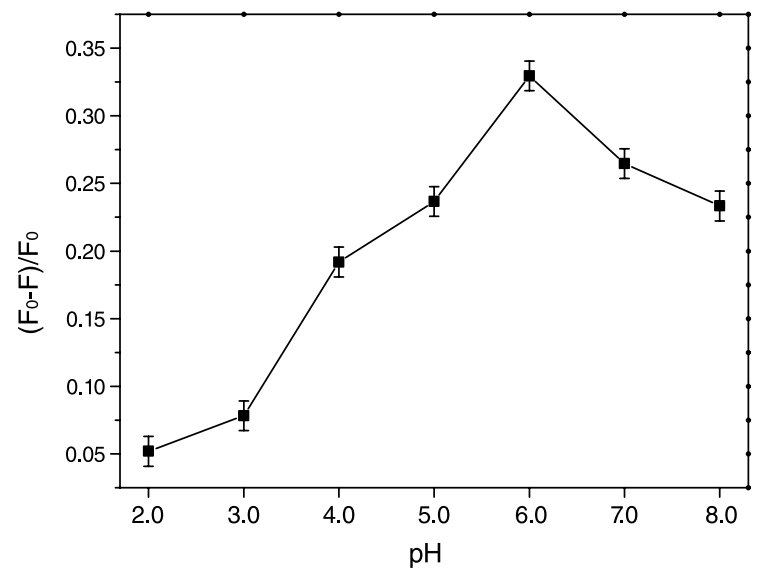

Figure 8. Effect of $\mathrm{pH}$ on the fluorescence intensity of agaric-based N-CQDs.

To determine the quenching response time of 2,4-DNP to agaric-based N-CQDs, a research has also been made on the reaction time. Figure 9 shows the change over time of fluorescence intensity of agaric-based N-CQDs after addition of 2,4-DNP. It can be seen that the fluorescence intensity drops sharply within 0-1 min, reaches its minimum at $1 \mathrm{~min}$ and gradually gets stable afterwards. Therefore, the reaction time of $1 \mathrm{~min}$ is defined as the optimal fluorescence quenching response time.

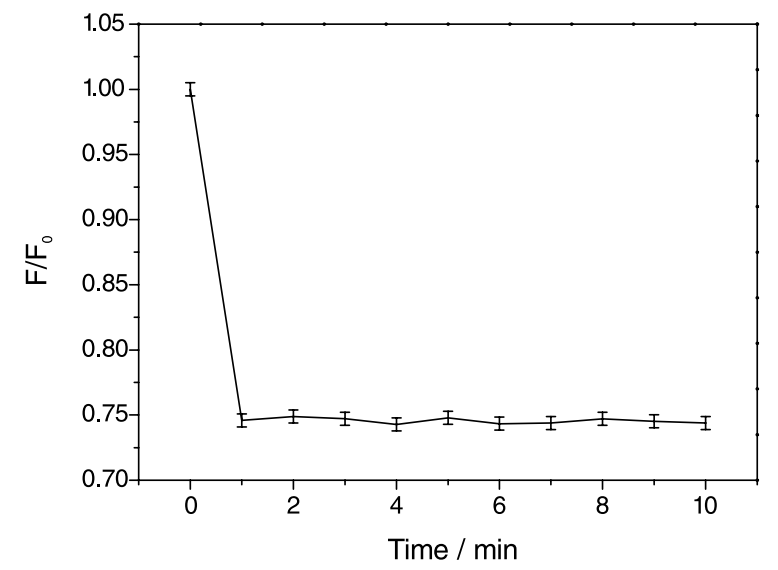

Figure 9. Effect of reaction time on the fluorescence intensity of agaricbased N-CQDs.

\section{Method performance study}

Under the optimal experimental conditions, the relationship between the fluorescence quenching intensity of agaric-based N-CQDs and the concentration of 2,4-DNP has been studied and the method for quantitative analysis and detection of fluorescence quenching of 2,4-DNP has been established. As shown in Figure 10, the quenching intensity of N-CQDs decreases gradually with the increase of the concentration of 2,4-DNP and presents regular changes, further demonstrating the feasibility and application prospective of N-CQDs to be used as fluorescence probe for detection of 2,4-DNP. As shown in Figure 11, the fluorescence quenching efficiency (F0/F) and the concentration of 2,4-DNP have a good linear relationship within $0.1-50 \mu \mathrm{M}$, the linear equations being $\mathrm{F} 0 / \mathrm{F}=0.8854+0.0811 \mathrm{C}(2,4-\mathrm{DNP})$, correlation coefficient $\left(\mathrm{R}^{2}=0.9969\right)$, and the limit of detection is $15.78 \mathrm{nM}$ (signalto-noise ratio $(\mathrm{S} / \mathrm{N})=3)$. It can be seen from comparative analysis that the agaric-based N-CQDs synthesized by this method show high sensitivity in the detection of 2,4-DNP and can be used for qualitative and quantitative analysis and detection of 2,4-DNP in practical samples.

\section{Analysis of fluorescence quenching mechanism}

With the addition of 2,4-DNP into the solution of CQDs, fluorescence quenching occurs, of which the mechanism 


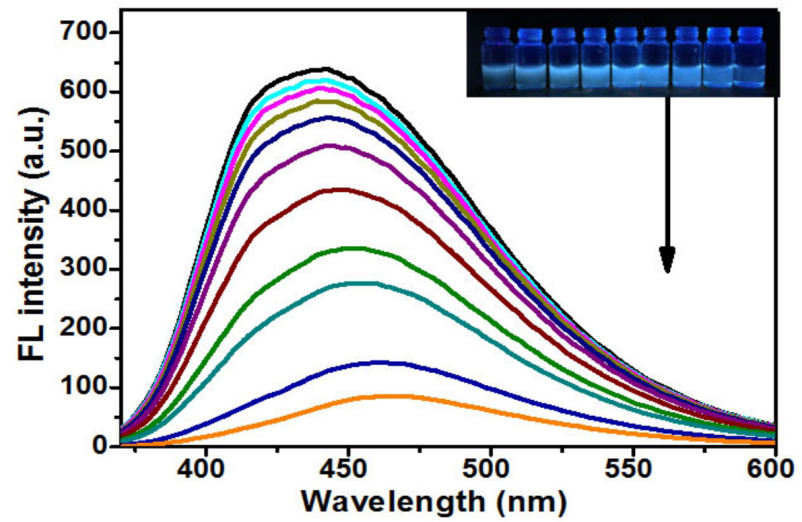

Figure 10. Fluorescence spectra of agaric-based N-CQDs upon addition of different concentrations of 2,4-DNP.

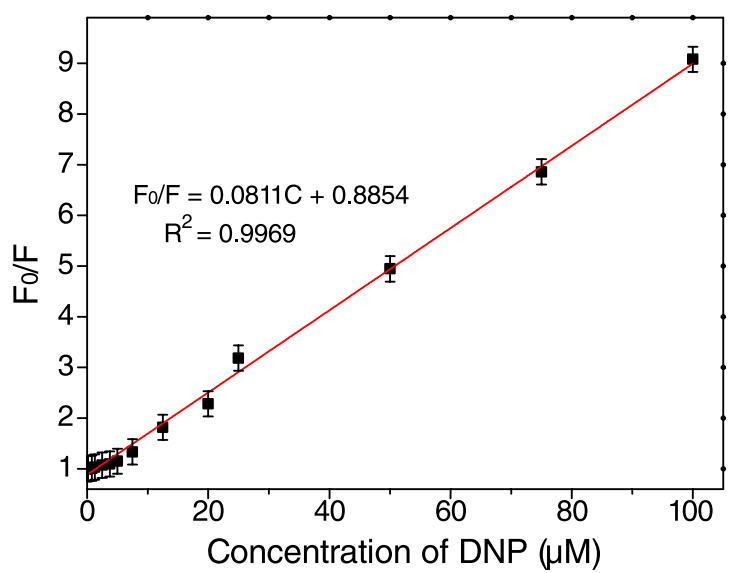

Figure 11. Relation curve of the fluorescence quenching efficiency (F0/F) versus the concentration of 2,4-DNP over the range from 0.1 to $50 \mu \mathrm{M}$.

may be the transfer of resonance energy. The absorption spectrum of 2,4-DNP falls within 360-440 nm, partly overlapping with the emission spectrum of CQDs. Besides, the mechanism of 4-nitrophenol quenching the fluorescence of CQDs has been reported in the literature. ${ }^{32}$ It is therefore concluded that transfer of fluorescence resonance energy occurs when the fluorescence of CQDs is quenched by 2,4-DNP.

\section{Detection of practical samples}

To verify the practicability of this method, the content of 2,4-DNP has been determined in different water samples. As shown in Table 1, the spike recoveries of water samples fall within 96.0-104.0\% and no relative standard deviation (RSD) is higher than $3.08 \%$. Therefore, this method is applicable to rapid analysis and detection of 2,4-DNP in environmental water samples and can be effectively applied to further research. This proposed procedure is comparable to or even better than the previous reports for 2,4-DNP detection (see Table 2).

\section{Bioluminescence imaging}

Bioluminescence imaging is a process where fluorescence reporter group, including inorganic materials (upconversion material, quantum dot, etc.) and organic materials (green fluorescent protein, red fluorescent protein, fluorescent dye, etc.), is used for labeling, and when reaching a high molecular energy level under exciting light, the reporter group will emit visible light with longer wavelength to form the interior bioluminescent source for detection. At present, the frequently-used fluorophores include various micromolecular fluorescent dyes, green fluorescent proteins and red fluorescent proteins. In recent years, fluorescence techniques have been widely applied to molecular biology study and study on metabolism of small molecules. As an important indicator of fluorescent nanomaterials in bioimaging, the cytotoxicity has

Table 1. Analytical results of 2,4-DNP in water samples $(n=3)$

\begin{tabular}{lccccc}
\hline Water sample & $\begin{array}{c}\text { Actual determination } \\
\text { value } /(\mu \mathrm{g} \mathrm{mL}-1)\end{array}$ & RSD / \% & $\begin{array}{c}\text { Spiked amount / } \\
\left(\mu \mathrm{g} \mathrm{mL} \mathrm{mL}^{-1}\right)\end{array}$ & $\begin{array}{c}\text { Measured value / } \\
\left(\mu \mathrm{g} \mathrm{mL} \mathrm{m}^{-1}\right)\end{array}$ & Recovery / \% \\
\hline Running water & 0.0132 & 2.34 & 5 & 5.094 & 102.0 \\
Water from Laoyuhe River & 0.0446 & 2.68 & 10 & 9.602 & 96.02 \\
Domestic wastewater & 0.0237 & 3.08 & 15 & 15.60 & 104.0 \\
\hline
\end{tabular}

RSD: relative standard deviation.

Table 2. Comparison of different optical CQDs for 2,4-DNP determination

\begin{tabular}{lcccc}
\hline Precursor & Linear range $/ \mu \mathrm{M}$ & LOD & RSD / \% & Reference \\
\hline Acetone & - & $0.4 \mathrm{mg} \mathrm{L}^{-1}$ & 1.7 & 33 \\
Ampicillin sodium & $0-25$ & $13.44 \mathrm{nM}$ & 1.23 & 34 \\
Palm shell powder and trifflic acid & $0.5-85$ & $165 \mathrm{nM}$ & - & 35 \\
Caramel & $0.4-35$ & $140 \mathrm{nM}$ & $\leq 5.2$ & 36 \\
Agarics & $0.1-50$ & $15.7 \mathrm{nM}$ & $\leq 3.08$ & this work \\
\hline
\end{tabular}

LOD: limit of detection; RSD: relative standard deviation. 
been first tested by 3-(4,5)-dimethylthiahiazo(-z-y1)3,5-di-phenytetrazoliumromide (MTT) for agaric-based $\mathrm{N}-\mathrm{CQD}$. The test results show that the cell viability is above $90 \%$ under the action of agaric-based N-CQDs with different concentrations, indicating a low cytotoxicity and thus a potential thereof to be applied to imaging of cell tissue. On this basis, agaric-based N-CQDs have been used in the study on imaging of HT29 cells. As shown in Figure 12, after incubation of HT29 cells with agaric-based $\mathrm{N}-\mathrm{CQD}$, blue, green and red fluorescence can be observed through laser scanning confocal microscope at excitation wavelength of 405, 488 and $552 \mathrm{~nm}$, respectively. It can thus be concluded that agaric-based N-CQDs can be used in fluorescence imaging in living cells.

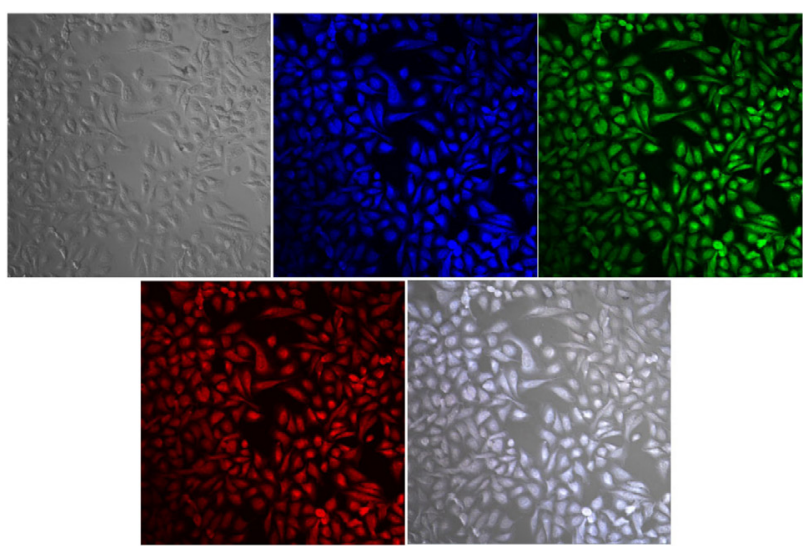

Figure 12. Confocal fluorescence images of HT29 cells excited under bright field, blue, green and red when excited at 405, 488 and $552 \mathrm{~nm}$, and overlay of bright field and red, respectively using the agaric based N-CQDs.

The toxicity to bacteria of agaric-based N-CQDs has also been evaluated. It has been found that after incubation of four unused living/dead bacteria, i.e., Colibacillus, Staphylococcus aureus, Bacillus and Listeria, in the solution of agaric-based N-CQDs for a certain period of time, only weak fluorescence or even no fluorescence can be observed from these bacteria except Bacillus from which clear imaging has been obtained. As shown in Figure 13, blue, green and red fluorescence can be observed at excitation wavelength of 405, 488 and $552 \mathrm{~nm}$, respectively. It can thus be concluded that agaric-based N-CQDs can be used in bioimaging due to a good inhibitory effect on but low toxicity to bacteria.

\section{Conclusions}

With agaric as a carbonaceous precursor and urea as nitrogen source, the N-CQDs with fluorescence quantum yield of $30.2 \%$ and excellent optical performance have been synthesized by the simple one-step hydrothermal
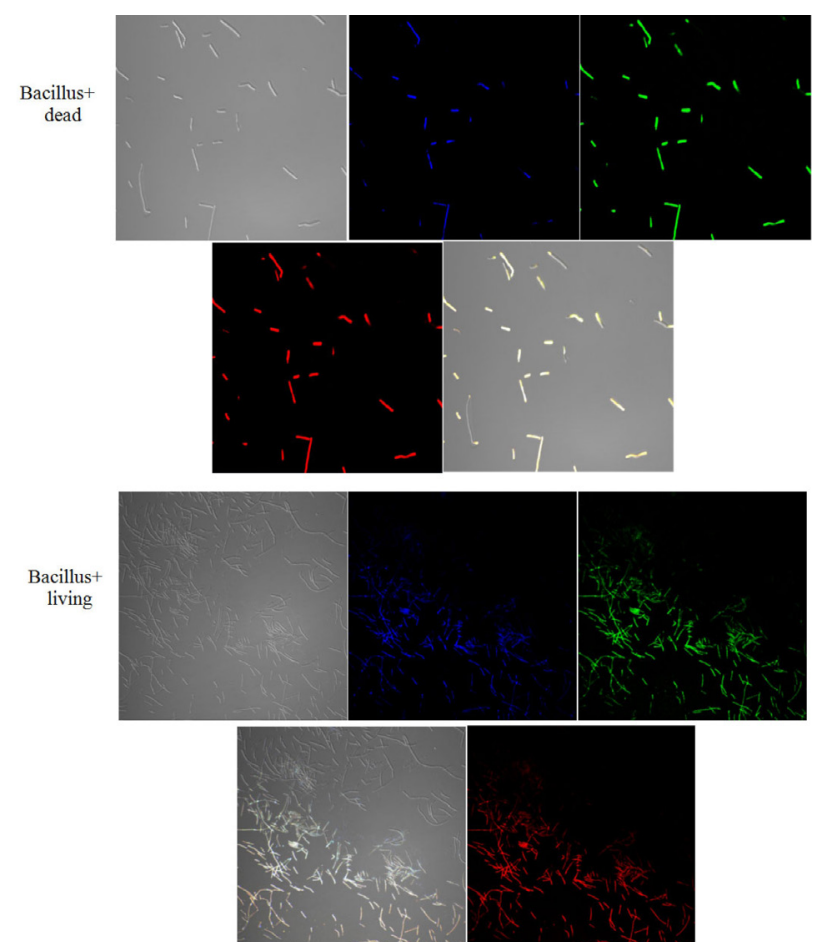

Figure 13. Fluorescence imaging of living/dead positive Bacillus colored by agaric-based N-CQDs.

method and characterized in terms of morphology, particle size, crystal structure, elementary composition and optical property by means of TEM, FTIR, XPS, UV-Vis and FS. The results show that the agaric-based $\mathrm{N}-\mathrm{CQD}$ s have uniform particle size distribution, abundant oxygen and nitrogen-bearing functional groups on the surface, and high reliance on excitation wavelength and good tunability. Agaric-based N-CQDs can be used as fluorescent nanoprobe in analysis and detection of 2,4DNP due to high sensitivity, high selectivity and strong disturbance resistance to 2,4-DNP based on the quenching mechanism of inner filter effect of fluorescence. With a good linear relation and characterized by rapid response, simple and convenient operation, and high sensitivity, good selectivity and low limit of detection, this method provides a new idea for detecting 2,4-DNP with CQDs as fluorescent nanoprobe and has a broad application prospect. It can thus be concluded that agaric-based $\mathrm{N}-\mathrm{CQDs}$ can be used in fluorescence imaging in living cells.

\section{Acknowledgments}

The work was greatly supported by Joint project of local universities-Youth project (No.202001BA070001-226), Joint project of local universities-General Project (No.202001BA070001-218), Yunnan Local Colleges Applied Basic Research Projects (No.2018FH001-054), 
Talent Introduction Project of Kunming University (No. YJL19006) (No.YJL20013).

\section{Author Contributions}

Yujiao Tu was responsible for methodology, cell bioimaging; Xianhao Long for investigation, validation, software; Zhihua Li, Jiao Zhao for data curation, writingreviewing and editing.

\section{References}

1. Phillips, L.; Singer, M. A.; Neurology 2013, 80, 773.

2. Jiang, J.; Yuan, Z.; Huang, W.; Wang, J.; Toxicol. Ind. Health 2011, 27, 323.

3. Grundlingh, J.; Dargan, P. I.; El-Zanfaly, M.; Wood, D. M.; J. Med. Toxicol. 2011, 7, 205.

4. Loos, R.; Tavazzi, S.; Paracchini, B.; Canuti, E.; Weissteiner, C.; Anal. Bioanal. Chem. 2013, 405, 5875.

5. Rahimi, A.; Hashemi, P.; Badiei, A.; Arab, P.; Ghiasvand, A. R.; Anal. Chim. Acta 2011, 695, 58.

6. Liu, Y.; Zhu, L. H.; Zhang, Y. Y.; Tang, H. Q.; Sens. Actuators, B 2012, 171, 1151.

7. Wang, J.; Wang, C. F.; Chen, S.; Angew. Chem., Int. Ed. 2012, $51,9297$.

8. Li, H.; Kang, Z.; Liu, Y.; Lee, S. T.; J. Mater. Chem. 2012, 22, 24230.

9. Li, H.; He, X.; Kang, Z.; Huang, H.; Liu, Y.; Liu, J.; Lian, S.; Tsang, C. H. A.; Yang, X.; Lee, S. T.; Angew. Chem. 2010, 49, 4430 .

10. Yin, B.; Deng, J.; Peng, X.; Long, Q.; Zhao, J.; Lu, Q.; Chen, Q.; Li, H.; Tang, H.; Zhang, Y.; Yao, S.; Analyst 2013, 138, 6551.

11. Qu, S.; Wang, X.; Lu, Q.; Liu, X.; Wang, L.; Angew. Chem., Int. Ed. 2012, 51, 12215.

12. Wang, J.; Qiu, J.; J. Mater. Sci. 2016, 51, 4728.

13. Yang, X.; Zhuo, Y.; Zhu, S.; Luo, Y.; Feng, Y.; Dou, Y.; Biosens. Bioelectron. 2014, 60, 292.

14. Goh, E. J.; Kim, K. S.; Kim, Y. R.; Jung, H. S.; Beack, S.; Kong, W. H.; Scarcelli, G.; Yun, S. H.; Hahn, S. K.; Biomacromolecules 2012, 13, 2554.

15. Cao, L.; Wang, X.; Meziani, M. J.; Lu, F.; Wang, H.; Luo, P. G.; Lin, Y.; Harruff, B. A.; Veca, L. M.; Murray, D.; Xie, S. Y.; Sun, Y. P.; J. Am. Chem. Soc. 2007, 129, 11318.

16. Lai, C. W.; Hsiao, Y. H.; Peng, Y. K.; Chou, P. T.; J. Mater. Chem. 2012, 22, 14403.
17. Algarra, M.; Campos, B. B.; Radotic, K.; Mutavdzic, D.; Bandosz, T.; Jiménez-Jiménez, J.; Rodriguez-Castellón, E.; da Silva, J. C. G. E.; J. Mater. Chem. A 2014, 2, 8342.

18. Lu, W.; Qin, X.; Liu, S.; Chang, G.; Zhang, Y.; Luo, Y.; Asiri, A. M.; Al-Youbi, A. O.; Sun, X.; Anal. Chem. 2012, 84, 5351.

19. Liu, J. M.; Lin, L. P.; Wang, X. X.; Lin, S. Q.; Cai, W. L.; Zhang, L. H.; Zheng, Z. Y.; Analyst 2012, 137, 2637.

20. Zhou, L.; Lin, Y.; Huang, Z.; Ren, J.; Qu, X.; Chem. Commun. 2012, 48, 1147.

21. Lin, Z.; Xue, W.; Chen, H.; Lin, J. M.; Anal. Chem. 2011, 83, 8245 .

22. Zhao, H. X.; Liu, L. Q.; Liu, Z. D.; Wang, Y.; Zhao, X. J.; Huang, C. Z.; Chem. Commun. 2011, 47, 2604.

23. Dong, Y.; Wang, R.; Tian, W.; Chi, Y.; Chen, G.; RSC Adv. 2014, 4, 3701.

24. Zhu, A.; Luo, Z.; Ding, C.; Li, B.; Zhou, S.; Wang, R.; Tian, Y.; Analyst 2014, 139, 1945.

25. Shi, W.; Wang, Q.; Long, Y.; Cheng, Z.; Chen, S.; Zheng, H.; Huang, Y.; Chem. Commun. 2011, 47, 6695.

26. Huang, Q.; Zhang, H.; Hu, S.; Li, F.; Weng, W.; Chen, J.; Wang, Q.; He, Y.; Zhang, W.; Bao, X.; Biosens. Bioelectron. 2014, 52, 277.

27. Xu, B.; Zhao, C.; Wei, W.; Ren, J.; Miyoshi, D.; Sugimoto, N.; $\mathrm{Qu}, \mathrm{X}$; Analyst 2012, 137, 5483.

28. Qian, Z.; Ma, J.; Shan, X.; Feng, H.; Shao, L.; Chem. - Eur. J. 2014, 20, 2254.

29. Dai, H.; Shi, Y.; Wang, Y.; Sun, Y.; Hu, J.; Ni, P.; Li, Z.; Sens. Actuators, B 2014, 202, 201.

30. Yang, F.; Wang, L.; Guo, Z.; Acta Chim. Sin. 2012, 70, 1283.

31. Vaz, R.; Bettini, J.; Júnior, J. G. F.; Lima, E. D. S.; Botero, W. G.; Santos, J. C. C.; Schiavon, M. A.; J. Photochem. Photobiol., A 2017, 346, 502.

32. Ahmed, G. H. G.; Laino, R. B.; Calzon, J. A. G.; García, M. E. D.; Microchim. Acta 2015, 182, 51.

33. Cayuela, A.; Soriano, M. L.; Valcárcel, M.; Anal. Chim. Acta 2013, 804, 246.

34. Yuan, X.; Tu, Y.; Chen, W.; Xu, Z.; Wei, Y.; Qin, K.; Zhang, Q.; Xiang, Y.; Zhang, H.; Ji, X.; Dyes Pigm. 2020, 175, 108187.

35. Harnish, S.; Padmaja, S.; Mater. Res. Bull. 2018, 108, 250.

36. Ma, J.; Bian, L.; Zhao, L.; Feng, X.; Zhao, L.; Wang, Z.; Pu, Q.; Talanta 2019, 197, 159.

Submitted: April 14, 2021

Published online: August 3, 2021 